\title{
The Puzzling Dynamos of Stars: Recent Progress With Global Numerical Simulations
}

\author{
Antoine Strugarek ${ }^{1,2}$, Patrice Beaudoin ${ }^{2}$, Paul Charbonneau ${ }^{2}$ and \\ Allan S. Brun ${ }^{1}$ \\ ${ }^{1}$ Laboratoire AIM Paris-Saclay, \\ CEA/Irfu Université Paris-Diderot CNRS/INSU, \\ F- 91191 Gif-sur-Yvette \\ email: antoine.strugarek@cea.fr \\ ${ }^{2}$ Département de physique, Université de Montréal, \\ C.P. 6128 Succ. Centre-Ville, Montréal, QC H3C-3J7, Canada
}

\begin{abstract}
The origin of magnetic cycles in the Sun and other cool stars is one of the great theoretical challenge in stellar astrophysics that still resists our understanding. Ab-initio numerical simulations are today required to explore the extreme turbulent regime in which stars operate and sustain their large-scale, cyclic magnetic field. We report in this work on recent progresses made with high performance numerical simulations of global turbulent convective envelopes. We rapidly review previous prominent results from numerical simulations, and present for the first time a series of turbulent, global simulations producing regular magnetic cycles whose period varies systematically with the convective envelope parameters (rotation rate, convective luminosity). We find that the fundamentally non-linear character of the dynamo simulated in this work leads the magnetic cycle period to be inversely proportional to the Rossby number. These results promote an original interpretation of stellar magnetic cycles, and could help reconcile the cyclic behaviour of the Sun and other solar-type stars.
\end{abstract}

Keywords. Sun: magnetic fields, Sun: interior, stars: magnetic fields, (magnetohydrodynamics:) MHD

\section{Introduction}

Cool stars such as the Sun possess a convective envelope that is thought to be at the origin of their internal large-scale flows (differential rotation, see Brun \& Toomre 2002, meridional circulation, see Featherstone \& Miesch 2015) and dynamic magnetic fields (see, e.g. Brun et al. 2015). Many possible mechanisms sustaining a dynamo in a convective envelope have been invoked in the literature (see, e.g. the review Charbonneau 2010), most of which rely on some parametrization of the magnetohydrodynamical turbulence that animates the convective layer. The magnetism of solar-like stars hence is a formidable theoretical challenge due to the extreme parameter regime in which interiors of stars operate. The magnetic field of a star furthermore plays a crucial role during its life, by shaping its wind and astrosphere (Cranmer 2012), determining its rotational braking (Réville et al. 2015), and even affecting its (exo)planets habitability (Lammer et al. 2009; Gallet et al. 2016).

In particular, the cyclic aspect of solar magnetism (and stellar magnetism, see e.g. Noyes et al. 1984; Baliunas et al. 1995; Saar \& Brandenburg 1999; Bohm Vitense 2007; Egeland et al. 2015; Metcalfe et al. 2016) remains as of today one of the great mysteries of stellar astrophysics. Indeed, if the dynamo process sustaining the large-scale cyclic magnetic field of the Sun is rooted in the convective turbulence itself, how can a characteristic time-scale of eleven years emerge since all hydrodynamical time-scales are 
significantly different? In other words, what sets the magnetic cycle period of the Sun and of other cool stars? Observational constraints on the magnetic cycle of distant stars are difficult to acquire because they require repeated monitoring of stars over long (at least decadal) periods of time. As a result, only a few observational programs (see previously cited efforts) have as of today been able to provide very useful observations of magnetic cycles in stars different than the Sun. Numerical simulations of stars hence provide as of today promising laboratories to explore the possible drivers of cyclic magnetic activity in stars.

In the past decade, significant progress has been made in understanding the sustainment of large-scale magnetic fields in turbulent convection zones of stars thanks to global, 3D numerical simulations. Only a handful simulations, though, present today a cyclic behaviour. We quickly review the current state of the art for modelling magnetic cycles in stars with global turbulent simulations in Section 2 (the reader may find a more detailed review in the Section 2.3 of Brun et al. 2015). In Section 3 and 4 we will present for the first time a series of numerical simulations exhibiting a cyclic behaviour, with a period that systematically varies with the rotation and luminosity of the modelled star.

\section{Cycles in global turbulent numerical simulations}

Large-scale magnetic fields in turbulent global numerical simulations. The basis of 3D global modelling of stellar interior flows and magnetism was first developed in the pioneering work of Gilman \& Miller (1981); Gilman (1983); Glatzmaier (1984, 1985); Brun et al. (2004). At that time, global scale magnetism was already achieved in turbulent simulations using enhanced diffusivity coefficients (which are still widely used today) to model the contribution of the scales unresolved by the numerical model. In those models the large-scale field was nonetheless less energetic than the small scale magnetic field, which is ubiquitously produced by the turbulent convective motions under the influence of rotation as long as the magnetic Reynolds number exceeds the critical onset of dynamo action. Many teams have since then attempted to carry out such simulations, mainly in the solar/stellar physics and planetary dynamo communities (a convective dynamo benchmark conducted by Jones et al. 2011). One very intriguing regime was in particular discovered by Brown et al. (2010), in which persistent wreaths of magnetic field are sustained near the bottom of the convection zone, inside the turbulent region itself.

Reversing magnetic fields in turbulent global numerical simulations. Many simulations then started to show quasi-regular magnetic field reversals, using the ASH code (Brown et al. 2011; Augustson et al. 2013; Nelson et al. 2013), the PENCIL code (Käpylä et al. 2013), the MagIC code (Gastine et al. 2012), or the PaRoDy code (see Schrinner et al. 2012 , 2014). In addition, evidence of buoyantly rising magnetic wreaths was found by Nelson et al. (2011). Finally, an almost cyclic activity was also found in simulations of the fully convective M-star Proxima Centauri by Yadav et al. (2016). In all these simulations, the large-scale magnetic field is observed to invert quasi-regularly on a yearly timescale, over a period of several hundreds of rotation periods of the star. Nevertheless, the detailed mechanism setting the exact inversion timing remains to be clarified as of today.

Cyclic magnetic fields in turbulent global numerical simulations. Only a handful of global turbulent simulations successfully produced truly regular magnetic cycles. The first solar-like cycles were arguably obtained by Ghizaru et al. (2010), using the EULAG code with an implicit large-eddy simulation approach (see Section 3.1). These results were subsequently analyzed (Beaudoin et al. 2013; Lawson et al. 2015) and compared to classical mean-field theory (Racine et al. 2011; Simard et al. 2013; Beaudoin et al. 
2016; Simard et al. 2016), revealing an $\alpha^{2}-\Omega$ like behaviour with significant departure from isotropic mean field $\alpha$ and $\beta$ tensors. In the meantime, Käpylä et al. (2012) also reported on cyclic solutions in global spherical wedges using the PENCIL code. In their solution, the cycle period was found to be much shorter (of the order of a few years), but presented a clear equatorial propagation of what would be equivalent to a solar activity band. In the simulations of Gastine et al. (2012), almost cyclic solutions are reported but exhibit a polar propagation and tend to show strong hemispheric decoupling. Finally, Augustson et al. (2015) also found a cyclic solution (also with a short cycle-period of a few years) using a hybrid dynamic-Smagorinski approach as a sub-grid scale model. They interestingly found a cyclic solution showing self-consistently a long period of minimal activity, that may relate to epochs like the so-called Maunder minimum of the Sun. So it is now clear that 3D global simulations can yield cyclic magnetic behaviour but such solutions are quite sensitive to the global parameters of the simulations, making it impossible to study their properties through an exploration of the parameter space and to deduce systematic trends.

\section{An implicit large-eddy simulation of a convection zone exhibiting cyclic magnetism}

\subsection{Numerical model}

The numerical simulations presented in this work are based on the hydrodynamical simulations presented in Strugarek et al. (2016). They consist in a spherical shell with a solar-like aspect ratio $\left(R_{\text {bottom }}=0.7 R_{\text {top }}\right)$ subject to a convective instability. The EULAG code (Smolarkiewicz \& Charbonneau 2013) is used to solve the Lipps-Helmer set of ideal MHD equations, written in the stellar rotating frame $\boldsymbol{\Omega}_{\star}$ as

$$
\begin{aligned}
\nabla \cdot(\bar{\rho} \mathbf{u}) & =0, \\
\mathrm{D}_{t} \mathbf{u} & =-\nabla\left(\frac{p}{\bar{\rho}}\right)-\frac{S}{c_{p}} \mathbf{g}-2 \mathbf{\Omega}_{\star} \times \mathbf{u}, \\
\mathrm{D}_{t} S & =-(\mathbf{u} \cdot \nabla) S_{a}-\frac{S}{\tau}, \\
\mathrm{D}_{t} \mathbf{B} & =(\mathbf{B} \cdot \nabla) \mathbf{u}-\mathbf{B}(\nabla \cdot \mathbf{u}) .
\end{aligned}
$$

In these anelastic equations the variables are perturbed quantities around a background isentropic state denoted with bars. In addition, an ambient hydrostatic state, denoted with the subscript ${ }_{a}$, was substracted from the equations. $\mathrm{D}_{t}$ is the material derivative. We recall that we use standard notation for the basic fluid quantities, i.e. $\mathbf{u}$ is the fluid velocity, $\rho$ its density, $p$ its pressure, $S$ its specific entropy, $\mathbf{B}$ the magnetic field, and $c_{p}=3.410^{8} \mathrm{erg} / \mathrm{g} / \mathrm{K}$ the specific heat at constant pressure. The background state is chosen to cover 3.22 density scale heights, which allows moderately small-scale convective structures at the top of the convective layer while retaining non-negligible stratification throughout the domain. The grid is held constant in all the simulations presented here, and is chosen relatively coarse $(51 \times 64 \times 128)$ to allow the exploration of decadal time-scale phenomena over a parameter space exploration.

As we consider a spherical, fully convective shell, boundary conditions are extremely important. The top and bottom boundaries are classically assumed to be stress-free walls. The magnetic boundary conditions are more delicate and were reported in equivalent setups (e.g. Brown et al. 2010) to significantly change the simulated dynamo. Here we use a radial field top boundary condition, to mimic the connection of the top of our convective envelope to a chromosphere and lower corona. At the bottom of our domain, we 
use a perfect conductor boundary condition to resemble the connection to an underlying conductive layer such as a stably stratified zone.

The convective instability is forced by the conjunction of the advection of the unstable ambient entropy profile $S_{a}$, and a Newtonian cooling term of characteristic timescale $\tau$ (for details, see Prusa et al. 2008; Smolarkiewicz \& Charbonneau 2013). The ambient entropy profile $S_{a}$ is defined in Strugarek et al. (2016) and is controlled by an entropy contrast $\Delta S$ throughout the modelled convective shell (in the model shown here, $\left.\Delta S \in[8,15] \times 10^{3} \mathrm{erg} / \mathrm{g} / \mathrm{K}\right)$. The Newtonian cooling in Equation 3.3 damps entropy perturbations over the timescale $\tau$ which is always chosen to exceed the convective overturning time. It ensures that on long time-scales, the model mimics a stellar convection zone remaining in thermal equilibrium (e.g. Cossette et al. 2016).

The resulting convective turbulence organizes such as to transport heat outward and is characterized by a convective luminosity calculated a posteriori and defined as

$$
L_{c}=4 \pi \bar{\rho} c_{P}\left\langle v_{r} T\right\rangle_{t, \varphi},
$$

where $T$ is the temperature perturbation and $c_{P}$ the specific heat at constant pressure, and \langle\rangle$_{t, \varphi}$ stands for the average over time and the azimuthal angle $\varphi$. Due to the particular convection forcing in our setup, and the existence of radial wall boundaries, the convective luminosity vary with radius in our models. Here we choose to estimate the convective luminosity $L_{b c}$ by averaging it over the $\left[0.75 R_{\odot}, 0.8 R_{\odot}\right]$, which is safely away from the lower boundary but close to the bottom part of the domain where the dynamo action primarily takes place (see below).

The EULAG code solves the ideal set of MHD equations, and as a result do not resolves any explicit dissipative process. Note that explicit dissipative process can be solved in the EULAG code, the results presented here nonetheless do not take them into account. The approach used here is a so-called implicit large-eddy simulation, in which the advective scheme MPDATA (Prusa et al. 2008; Smolarkiewicz \& Charbonneau 2013) adds up the necessary numerical dissipation to ensure the stability of the numerical scheme. This results in a very time- and space-dependant effective dissipation that occurs only in strong gradient regions. This approach has the advantage that the largest scales in the domain are saturated and sustained by inviscid non-linear processes as in real stellar convection zones, while the numerical dissipation acts mostly at small-scale. This scheme was shown to be compatible with classical turbulent cascades in 3D isotropic and homogeneous turbulence (Domaradzki et al. 2003) and with standard laplacian viscosity and heat diffusion (at small scale) in spherical convective shells (Strugarek et al. 2016).

\subsection{Cyclic magnetic fields in turbulent convection zones}

We consider a set of 7 numerical simulations covering about a factor of 2 in rotation rate and 3 in convective luminosity. After a transient phase of exponential growth of the magnetic field, the magnetic energy saturates in all the models at about $10 \%$ of the total kinetic energy.

We display the time evolution of the energies in a subset of three of our models in Figure 1. The three simulations exhibit a cyclic magnetic energy (black, 'ME') and the cyclic behaviour is found in both the mean toroidal (magenta, 'TME') and fluctuating (yellow, 'FME') components of the magnetic energy. The three magnetic energies oscillate in phase, and in these simulations the fluctuating magnetic energy dominates over the mean toroidal energy. The same cycle is also found in the differential rotation energy (green, 'DRKE', see also the differential rotation pattern on the right panels) and in the total kinetic energy (blue, 'KE'). Epochs of maximum DRKE correlate very well with minima of magnetic energy, suggesting an energy beating between the magnetic 

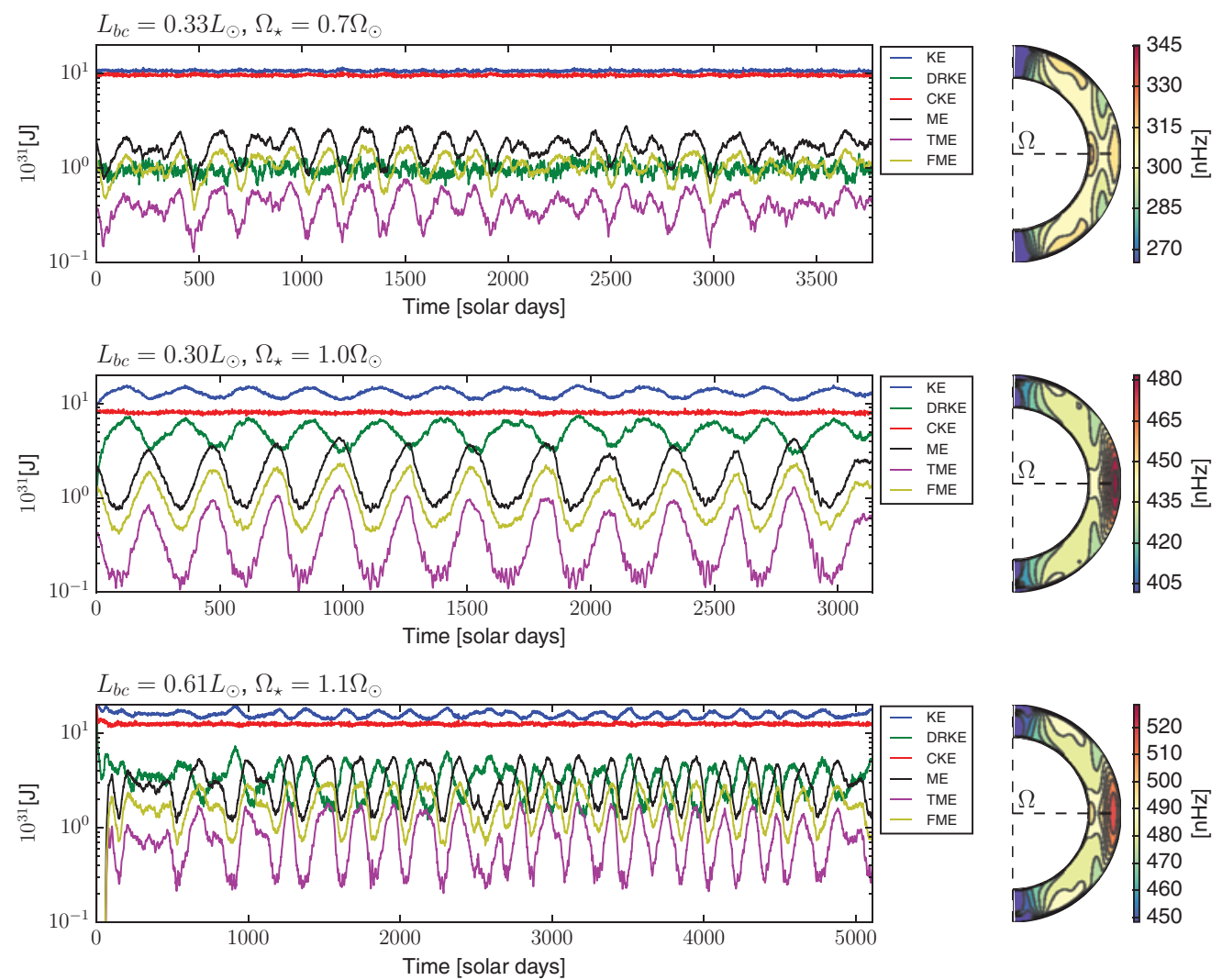

Figure 1. Magnetic cycles in three representative models. The left panels show energies as a function of time, integrated over the all convective envelope. The total kinetic energy (KE) is shown in blue, the differential rotation kinetic energy (DRKE) in green, and the convective kinetic energy (CKE) in red. The total magnetic energy (ME) is shown in black, the toroidal magnetic energy (TME) in magenta and the turbulent magnetic energy (FME) in yellow. The global parameters of the models (convective luminosity $L_{b c}$, rotation rate $\Omega_{\star}$ ) are indicated in each plot. The right panel show the differential rotation profile on the meridional plane, color-centered on the model rotation rate $\Omega$.

and differential rotation energy reservoirs along the observed cycles. Supporting this interpretation, the convective kinetic energy (red, 'CKE') does not show any significant modulation with the magnetic cycle, which suggests a dynamo scenario in which energy is exchanged between the large-scale differential rotation and the small and large-scale magnetic energy, and points to a significant back-reaction of the Lorentz force on the balance sustaining the large-scale differential rotation profile (right panels in Figure 1). Indeed, when the magnetic energy decreases the differential rotation is able to grow back until the magnetic back-reaction sets in again. The differential rotation profile remains solar-like (fast equator, slow poles) in the whole set of simulations presented here, but its amplitude and detailed latitudinal profile change significantly in our simulation set. As a result, the dynamo mechanism at the heart of these simulations seems to be robust with respect to the detailed profile of the differential rotation.

The azimuthal component of the cyclic large-scale magnetic field is shown in Figure 2 in a time-latitude diagram at depth $r=0.75 R_{\odot}$, near the bottom of our domain. Three snapshots of the azimutal magnetic field on the meridional plane are shown, showing the reversal of the large-scale field. The azimutal field is primarily located in the bottom half 

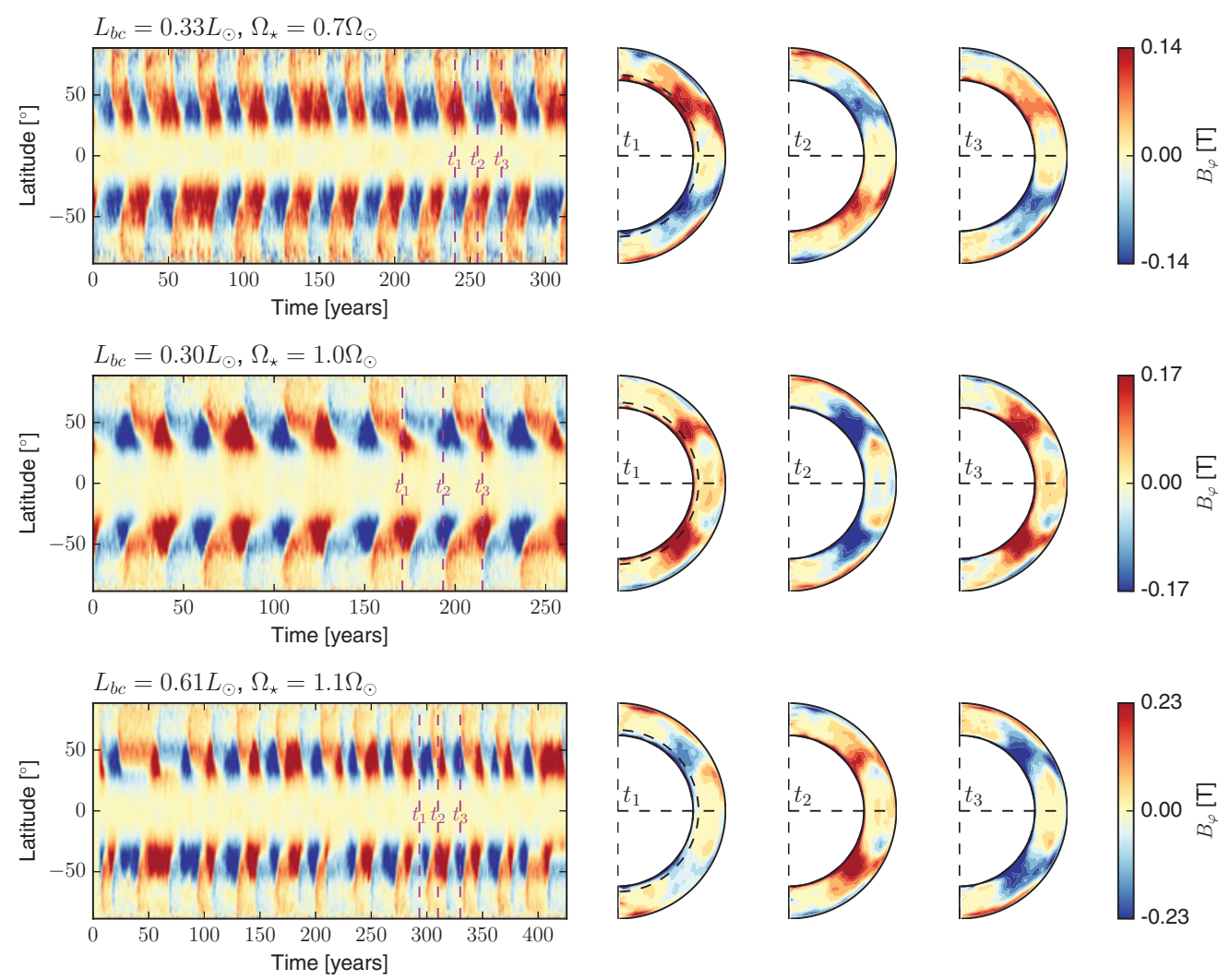

Figure 2. Azimuthal component of the mean magnetic field $\left\langle B_{\varphi}\right\rangle_{\varphi}$ in the same series of models as in Figure 1. $\left\langle B_{\varphi}\right\rangle_{\varphi}$ is shown in a time-latitude diagram at $r=0.75 R_{\odot}$ on the left panel. The three meridional plane snapshots in the right panels are taken at the time labeled by the dashed magenta lines.

of our convection zone and at mid-latitude, right outside of the inner tangent cylinder. A clear equatorial propagation of the azimuthal field is observed on the left panels, confined at mi-latitude near the maximum of the latitudinal shear. In all models, the magnetic field reaches a few tenths of a Tesla at the base of the convective envelope.

We readily see in Figure 2 that the large-scale azimuthal field is either symmetric (i.e. quadrupolar) or anti-symmetric (i.e. dipolar) with respect to the equator depending on the model and on the epoch of the model. We show in Figure 3 the dipolar/quadrupolar ratio at $R=0.75 R_{\odot}$ in the first model $\left(L_{\star}=0.33 L_{\odot}, \Omega=0.7 \Omega_{\odot}\right)$. The magnetic cycle clearly appears (over-plotted in grey in Figure 3), on top of which the large-scale field oscillates between periods of predominantly dipolar and predominantly quadrupolar geometries, with no clear regularity (longer time integrations would be required with this simulation to confirm this lack or regularity). The beating between the two topologies suggests a coupling between the dynamo families in our models (McFadden et al. 1991; Gubbins \& Zhang 1993; Knobloch et al. 1998), which is often a trace of a strongly nonlinear dynamo regime. In both situations, the dominant polarity is maximized (resp. minimized) during epochs of maxima (resp. minima) of magnetic energy, which differs from the situation observed in the Sun (DeRosa et al. 2012).

The dynamo acting in our simulations operates for differential rotations of various strength and profile (see the right panels of Figure 1). This warrants further 


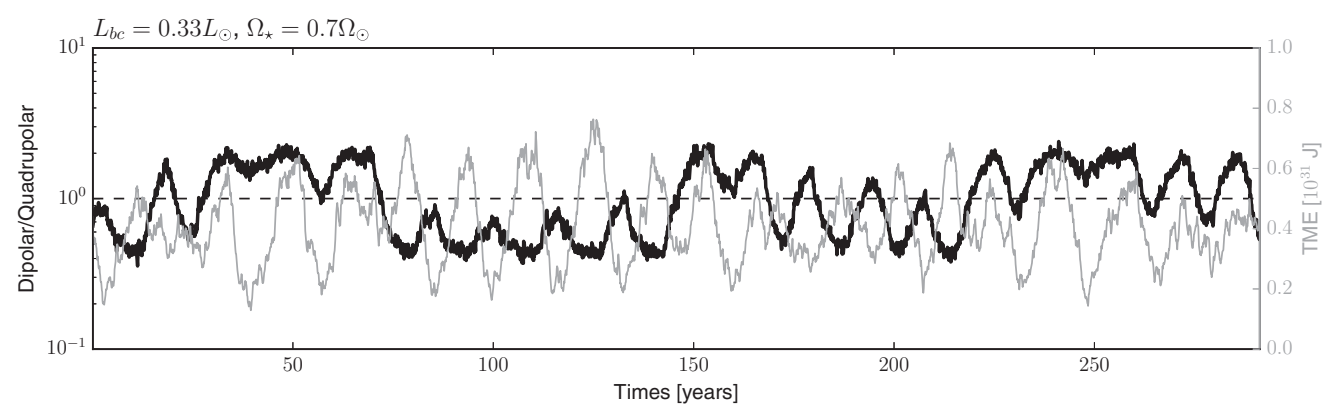

Figure 3. Dipolar/quadrupolar ratio (thick black line) as a function of time at $r=0.75 R_{\odot}$, for the first models shown in Figures 1 and 2. The volume-integrated toroidal magnetic energy (TME) is overlaid as a thin grey line.

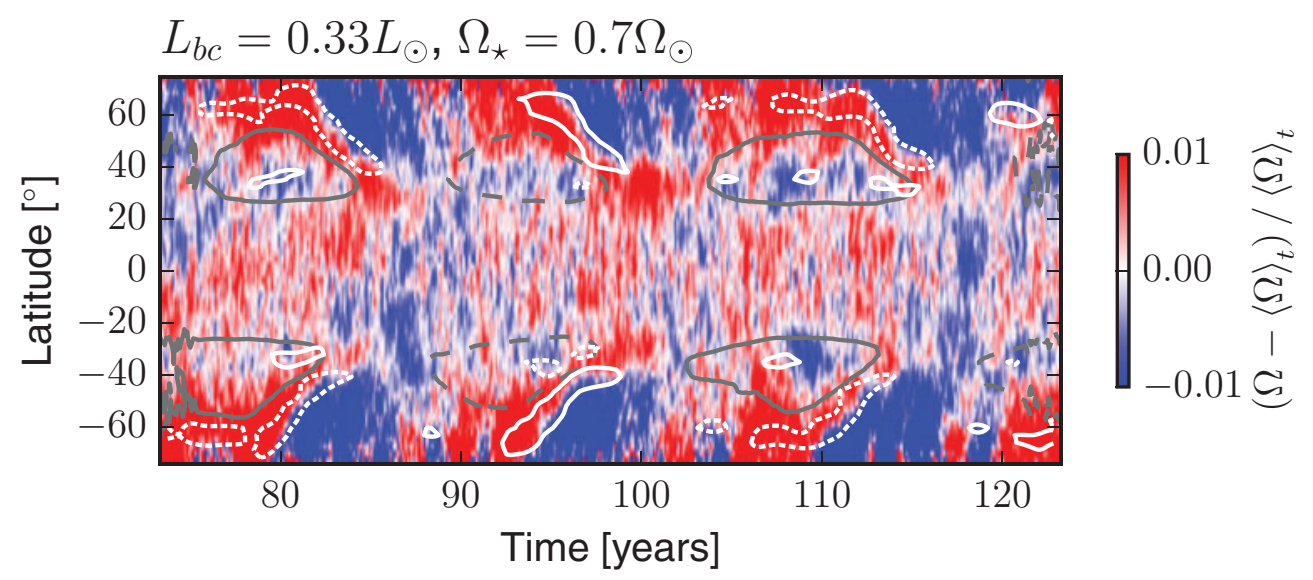

Figure 4. Toroidal field inversion in our modelled dynamo. The color map represents the temporal fluctuation of the differential rotation, defined as $\delta \Omega=\Omega-\langle\Omega\rangle_{t}$. Red tones denote an acceleration with respect to the mean differential rotation, while blue tones denotes a deceleration. The $[-0.1 \mathrm{~T}, 0.1 \mathrm{~T}]$ contours of $\left\langle B_{\varphi}\right\rangle_{\varphi}$ are overlaid in grey. Contours of the action of the differential rotation on the mean magnetic field are shown in white, and correlate very well with the cyclic destruction of $\left\langle B_{\varphi}\right\rangle_{\varphi}$. Positive contours are shown with solid lines, and negative contours with dashed lines.

investigation on the origin of the magnetic reversals in our models. We display in Figure 4 iso-contours of the azimuthal component of the mean magnetic field at $0.1 \mathrm{~T}$ in grey on a time-latitude diagram zoomed over a particular subset of cycles. We overplot the contribution of the differential rotation shear to the time-evolution of $\left\langle B_{\varphi}\right\rangle_{\varphi}$ as white contours (for all contours, plain line denote positive value and dashed lines negative values). We observe that the mean component of the induction associated with the mean azimuthal flow correlates very well with the destruction of the mean azimuthal magnetic field. The background colormap actually reveals the origin of the reversals. It shows the deviation from the mean differential rotation over time. Red threads originating at high latitudes and propagating poleward trace acceleration epochs in the differential rotation that trigger the polarity reversal of the mean magnetic field. These modulations of the differential rotation take their roots in the non-linear feedback of the magnetic field on the balance establishing the differential rotation through the Lorentz force. As result, the reversals observed in our simulations originate from a non-linear modulation of the differential rotation due to the presence of a large-scale magnetic field. Taken at face value, these 
considerations suggest a dynamo mechanism in which the cycle period, which is a tracer of the strength of dynamo action, is expected to decrease as the differential rotation weakens (since the non-linear magnetic feedback is then able to impact more efficiently the balance establishing the differential rotation). This is indeed what we observe, and we now turn to our full set of numerical simulations to determine what are the control parameters setting the cycle period in our non-linear, global dynamo.

\section{Modulation of the cycle period in global dynamo simulations}

We saw in Figure 2 that the cycle period of our simulated dynamos varies when the luminosity or the rotation rate of the model changes. In order to robustly estimate the cycle period in our models, we consider the azimuthally averaged azimuthal component of the magnetic field $b_{\varphi}(r, \theta, t)=\left\langle B_{\varphi}\right\rangle_{t, \varphi}$. At each $(r, \theta)$ point on the meridional plane, we compute the Fourier transform of $b_{\varphi}$. We automatically identify the peak in the Fourier spectrum, and calculate the width of the peak (defined as the width for which the Fourier spectrum decreases to $10 \%$ of its peak value) to be used as a proxy for an error bar. We then compute the probability density function of the cycle periods obtained for each $(r, \theta)$, and define the cycle period of the model as the peak in this distribution function. The error-bar is then also averaged over the $(r, \theta)$ points close to the peak cycle period.

We further define the fluid Rossby number in our models as

$$
R_{o f}=\frac{|\nabla \times \mathbf{u}|}{2 \Omega_{\star}} .
$$

We calculate the Rossby number $R_{o f}$ as function of radius and latitude, and define the average Rossby number $R_{0}$ inside the white wedge defined in the right panel of Figure 5 , which corresponds to the region where the dynamo action is strong (i.e. near the bottom of the convection zone, excluding the polar regions). We show on the left panel the cycle period realized in each of our seven models as a function of the Rossby number (in log scale). The cycle period is found to be inversely proportional to the Rossby number, $P_{\text {cyc }} \propto R_{o}^{-1.1 \pm 0.2}$. This result may seem counter-intuitive, as standard and fully linear $\alpha-\Omega$ dynamo models are expected to exhibit a cycle period which is directly proportional to the Rossby number (e.g. Noyes et al. 1984). This apparent contradiction is not so surprising, as we showed in Figure 4 that the dynamo operating in our set of simulations is different from the standard $\alpha-\Omega$ dynamo: temporal fluctuations of the large-scale differential rotation associated to the back-reaction from the Lorentz force play a dominant role in the polarity reversal. The dynamo at stake here is consequently fundamentally non-linear (i.e. non kinematic, as in Augustson et al. 2015), and subsequently exhibits a different scaling law compared to kinematic models of stellar dynamos. We are currently investigating in more details the exact dynamo process presented here, and in particular the dynamics of the poloidal field reversal, the role played by the small scale turbulence, and the effect of the cyclic modulations on the meridional circulation pattern (see also Beaudoin et al. 2013).

\section{Conclusions}

In this work we have shown for the very first time a series of global, 3D turbulent numerical simulations of stellar convective envelopes producing regular stellar magnetic cycles, which period varies with the rotation rate of the star and its convective luminosity. The dynamo sustaining the cyclic field is found to be fully non-linear and be able to generate large scale fields of both dipolar and quadrupolar families. The non-linearity of 

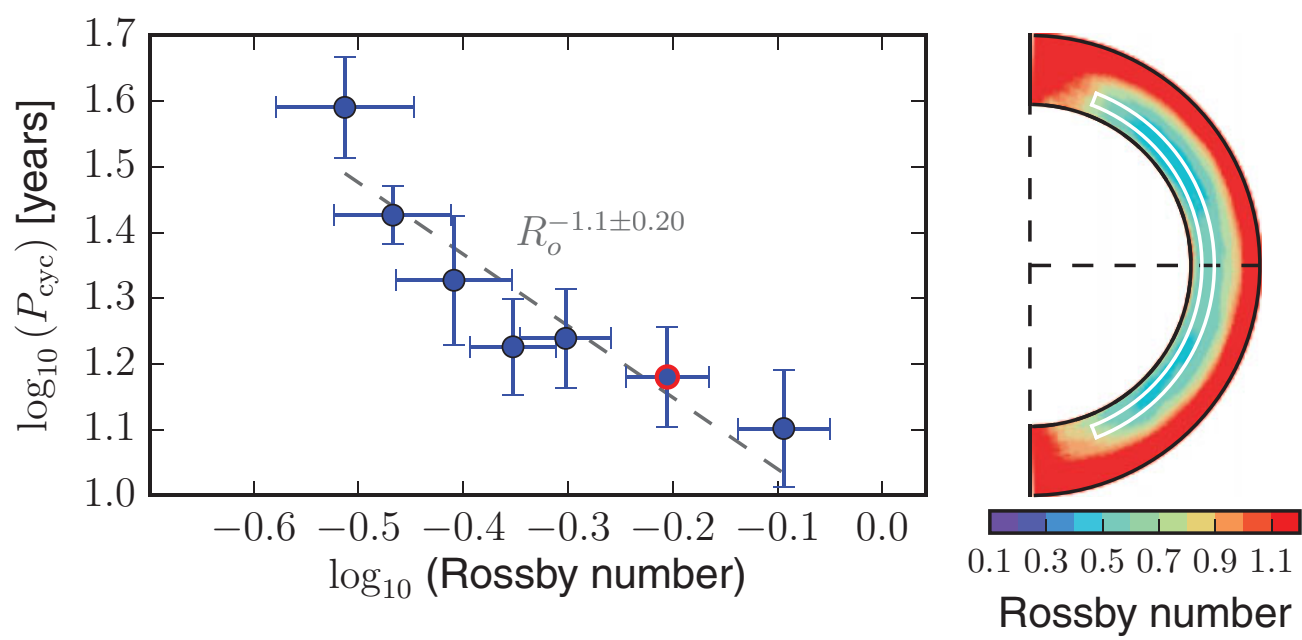

Figure 5. Left panel: cycle period as a function of the Rossby number in log scale. Right panel: Rossby number in the meridional plane of the model shown in Figures 3 and $4\left(L_{b c}=0.33 L_{\odot}\right.$, $\left.\Omega_{\star}=0.7 \Omega_{\odot}\right)$ and highlighted in red in the left panel. The white wedge labels the region in which the representative Rossby number of the simulation is evaluated.

the dynamo strikingly appears in the predominant role played by the temporal fluctuations of the differential rotation driven by the feedback of the magnetic field through the Lorentz force.

The boundary conditions, especially the lower magnetic boundary, play a dominant role in the dynamo state achieved in the simulations. Here we considered reasonable choices given the simplicity of our numerical setup. We also tested changing the lower magnetic boundary condition from perfect conductor to radial field, which completely changed the dynamo state and shut down the cycle. It seems that being able to store horizontal fields in an underlying region is henceforth important in developing a magnetic cycle, albeit the detailed fate of such a stored field appear not to matter in our set simulations.

We also explored various density contrasts $N_{\rho}$ (not shown here) from 2 to 4, which effectively changes the Rossby number of the simulated convective envelope. All these simulations fall on the same trend identified in Figure 5, namely the cycle period is found to be robustly inversely proportional to the Rossby number when changing the rotation rate, the luminosity, or the stratification of the modelled convection zone.

Several aspects require further investigations which are currently being pursued. Simulations at higher resolution are currently underway, in order to assess the robustness of the dynamo mechanism identified in our simulations with respect to the dissipative properties of the plasma. Furthermore, the complete dynamo loop still eludes our understanding and dedicated analysis are still required to fully assess the mode of operation of the dynamo in the simulations. Finally, we are also investigating twin simulations where a stably stratified zone is added to the bottom of the convective envelope to assess the importance of an underlying radiative zone in setting the cycle period (e.g. Browning et al. 2006; Lawson et al. 2015; Guerrero et al. 2016). Ultimately, we aim at reproducing the benchmarking exercise carried out in (see Strugarek et al. 2016) for the MHD models presented here, and evaluate the robustness of our dynamo mechanism for various small and sub-grid scale behaviours. The dynamo presented in this work opens new possibilities in interpreting the magnetic cycle of solar-like stars (Bohm Vitense 2007; Metcalfe et al. 
2016), and could help reinstate the Sun as a truly typical solar-type star (Salabert et al. 2016).

\section{References}

Augustson, K., Brun, A. S., Miesch, M., \& Toomre, J. 2015, ApJ, 809, 149

Augustson, K. C., Brun, A. S., \& Toomre, J. 2013, ApJ, 777, 153

Baliunas, S. L., Donahue, R. A., Soon, W. H., et al. 1995, ApJ, 438, 269

Beaudoin, P., Charbonneau, P., Racine, E., \& Smolarkiewicz, P. K. 2013, Sol. Phys., 282, 335

Beaudoin, P., Simard, C., Cossette, J.-F., \& Charbonneau, P. 2016, ApJ, 826, 138

Bohm Vitense, E. 2007, ApJ, 657, 486

Brown, B. P., Browning, M. K., Brun, A. S., Miesch, M. S., \& Toomre, J. 2010, ApJ, 711, 424

Brown, B. P., Miesch, M. S., Browning, M. K., Brun, A. S., \& Toomre, J. 2011, ApJ, 731, 69

Browning, M. K., Miesch, M. S., Brun, A. S., \& Toomre, J. 2006, ApJ, 648, L157

Brun, A. S., Garcia, R. A., Houdek, G., Nandy, D., \& Pinsonneault, M. 2015, Space Sci Rev, 196, 303

Brun, A. S., Miesch, M. S., \& Toomre, J. 2004, ApJ, 614, 1073

Brun, A. S. \& Toomre, J. 2002, ApJ, 570, 865

Charbonneau, P. 2010, LRSP, 7, 3

Cossette, J.-F., Charbonneau, P., Smolarkiewicz, P. K., \& Rast, M. P. 2016, Submitted to ApJ

Cranmer, S. R. 2012, Space Sci Rev, 172, 145

DeRosa, M. L., Brun, A. S., \& Hoeksema, J. T. 2012, ApJ, 757, 96

Domaradzki, J. A., Xiao, Z., \& Smolarkiewicz, P. K. 2003, PoF, 15, 3890

Egeland, R., Metcalfe, T. S., Hall, J. C., \& Henry, G. W. 2015, ApJ, 812, 12

Featherstone, N. A. \& Miesch, M. S. 2015, ApJ, 804, 67

Gallet, F., Charbonnel, C., Amard, L., et al. 2016, To appear in A\&A, 1608

Gastine, T., Duarte, L., \& Wicht, J. 2012, A\&\&A, 546, 19

Ghizaru, M., Charbonneau, P., \& Smolarkiewicz, P. K. 2010, ApJL, 715, L133

Gilman, P. A. 1983, ApJ Supp. Series, 53, 243

Gilman, P. A. \& Miller, J. 1981, ApJ Supp. Series, 46, 211

Glatzmaier, G. A. 1984, J. Comp. Phys., 55, 461

-. 1985, ApJ, 291, 300

Gubbins, D. \& Zhang, K. 1993, Physics of the Earth and Planetary Interiors, 75, 225

Guerrero, G., Smolarkiewicz, P. K., de Gouveia Dal Pino, E. M., Kosovichev, A. G., \& Mansour, N. N. 2016, ApJ, 819, 104

Jones, C. A., Boronski, P., Brun, A. S., et al. 2011, Icarus, 216, 120

Käpylä, P. J., Mantere, M. J., \& Brandenburg, A. 2012, ApJ, 755, L22

Käpylä, P. J., Mantere, M. J., Cole, E., Warnecke, J., \& Brandenburg, A. 2013, ApJ, 778, 41

Knobloch, E., Tobias, S. M., \& Weiss, N. O. 1998, MNRAS, 297, 1123

Lammer, H., Bredehöft, J. H., Coustenis, A., et al. 2009, The Astron. and Astrophys. Rev., 17, 181

Lawson, N., Strugarek, A., \& Charbonneau, P. 2015, ApJ, 813, 95

McFadden, P. L., Merrill, R. T., McElhinny, M. W., \& Lee, S. 1991, J. of Geophys. Res., 96, 3923

Metcalfe, T. S., Egeland, R., \& van Saders, J. 2016, ApJL, 826, L2

Nelson, N. J., Brown, B. P., Brun, A. S., Miesch, M. S., \& Toomre, J. 2011, ApJL, 739, L38

-. 2013, ApJ, 762, 73

Noyes, R. W., Weiss, N. O., \& Vaughan, A. H. 1984, ApJ, 287, 769

Prusa, J. M., Smolarkiewicz, P. K., \& Wyszogrodzki, A. A. 2008, Computers \& Fluids, 37, 1193

Racine, É., Charbonneau, P., Ghizaru, M., Bouchat, A., \& Smolarkiewicz, P. K. 2011, ApJ, 735, 46

Réville, V., Brun, A. S., Matt, S. P., Strugarek, A., \& Pinto, R. F. 2015, ApJ, 798, 116

Saar, S. H. \& Brandenburg, A. 1999, ApJ, 524, 295

Salabert, D., Garcia, R. A., Beck, P. G., et al. 2016, A\&A, 596, A31 
Schrinner, M., Petitdemange, L., \& Dormy, E. 2012, ApJ, 752, 121

Schrinner, M., Petitdemange, L., Raynaud, R., \& Dormy, E. 2014, A\&A, 564, A78

Simard, C., Charbonneau, P., \& Bouchat, A. 2013, ApJ, 768, 16

Simard, C., Charbonneau, P., \& Dubé, C. 2016, Adv. Spa. Res., 58, 1522

Smolarkiewicz, P. K. \& Charbonneau, P. 2013, J. Comp. Phys., 236, 608

Strugarek, A., Beaudoin, P., Brun, A. S., et al. 2016, Adv. Spa. Res., 58, 1538

Yadav, R. K., Christensen, U. R., Wolk, S. J., \& Poppenhaeger, K. 2016, To appear in A\&A, 1610.02721 